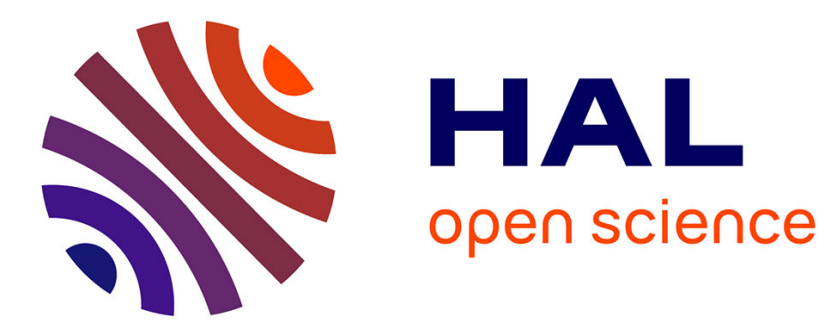

\title{
Peintures murales romaines de Famechon (Somme)
}

Alix Barbet, Didier Vermeersch

\section{To cite this version:}

Alix Barbet, Didier Vermeersch. Peintures murales romaines de Famechon (Somme). Gallia - Fouilles et monuments archéologiques en France métropolitaine, 1980, 38 (1), pp.117-135. 10.3406/galia.1980.1791. hal-01938971

\section{HAL Id: hal-01938971 \\ https://hal.science/hal-01938971}

Submitted on 27 Feb 2020

HAL is a multi-disciplinary open access archive for the deposit and dissemination of scientific research documents, whether they are published or not. The documents may come from teaching and research institutions in France or abroad, or from public or private research centers.
L'archive ouverte pluridisciplinaire $\mathbf{H A L}$, est destinée au dépôt et à la diffusion de documents scientifiques de niveau recherche, publiés ou non, émanant des établissements d'enseignement et de recherche français ou étrangers, des laboratoires publics ou privés.

\section{(이)(\$)}

Distributed under a Creative Commons Attribution - NonCommercial - NoDerivatives| 4.0 


\title{
PEINTURES MURALES ROMAINES DE FAMECHON (Somme)
}

\author{
par Alix BARBET et Didier VERMEERSCH
}

\section{I. Étude techNique et DEscriptive}

En 1972, l'exploitation d'une couche de silex dans le marais communal de Famechon révélait l'existence de constructions gallo-romaines le long de la petite rivière des Évoissons. Depuis, grâce à la bienveillance de M. Boulle, maire de la commune, le Groupe d'archéologie des étudiants d'Amiens mène une fouille de sauvetage qui a permis de reconnaître une villa de fond de vallée caractérisée par un long bassin ${ }^{1}$, comme celles de Mercin-et-Vaux et de Pont-d'Ancy (commune de Limé) dans l'Aisne?2.

Lors d'un sondage effectué en 1975 , l'exploitant de la ballastière mit au jour un dépotoir comblant l'ancien cours des Évoissons qui, auparavant, longeait la partie sud de la villa, puis fut déplacé vers le nord à l'époque romaine. C'est dans ce dépotoir de la fin du ir ${ }^{\mathrm{e}}$ siècle et du début du III siècle ap. J.-G. que furent trouvés les fragments de peintures murales que nous présentons ici. Ces derniers ont été jetés lors du détournement du cours des Évoissons, dans un moment où la villa était remaniée, et ont formé le fond du dépotoir. On peut dater ce remaniement au plus tard de la fin du $\mathrm{II}^{\mathrm{e}}$ siècle.

Nous avons rencontré de nombreux problèmes d'extraction : l'humidité constante et l'acidité de la terre avaient bien souvent rendu le mortier pulvérulent, et détaché la pellicule picturale de son support. Les peintures étaient très fragmentées et les morceaux dépassaient rarement 15 à $20 \mathrm{~cm}^{2}$, hormis une quinzaine de plaques de plus grandes dimensions. Elles furent recueillies avec soin, encollées à une toile de gaze par une fine couche de paraffine, selon les conseils d'Alix Barbet'. Étant donné l'état du terrain c'est la seule méthode qui se soit révélée efficace pour l'extraction de ces plaques et pour leur permettre

1 1). et E. Vermeersch, La villa de fond de vallée de Famechon (Somme), dans Bulletin de la Société des Antiquaires de Picardie, Amiens, $4^{\mathrm{e}}$ trimestre, 1976, p. 314-332 et J.-L. Cadolx, Informations archéologiques, dans Gallia, 35, 1977, p. 315-316.

$2 \quad$ Ibid., p. 297-298.

3 Alix Bariet, La peinlure murale romaine, Nolice technique du Touring Club de France, no 20, Paris, 1969 (') Bulletin de liaison du Centre d'Étude des Peintures Murales Romaines, no 1 (abrégé : C.E.P.M.R.). 

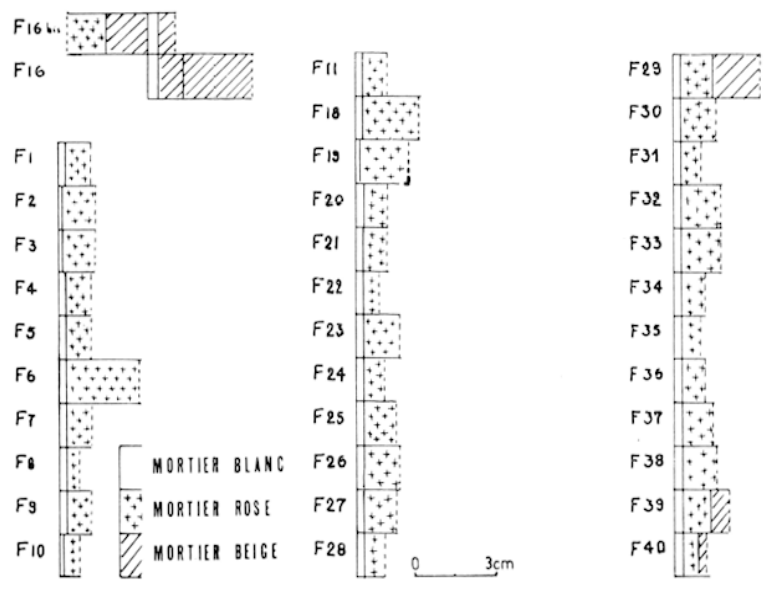

1 Jiagramme d'épaisseur des morliers.

d'attendre le nettoyage et le traitement de consolidation. Cette technique a été employée sans aucun inconvénient aussi bien sur la pellicule picturale que sur le revers de mortier.

En tout, c'est une quinzaine de caisses qui furent confiées au Centre d'étude des peintures murales romaines à Soissons ${ }^{4}$, où nous avons effectué un stage, pour suivre le déroulement des opérations. Après avoir été photographiées, toutes les plaques ont été débarrassées de leurs salissures au bistouri, ou bien, lorsque les concrétions étaient trop dures, à la mini-meuleuse. Une fois parfaitement propres, les peintures ont été protégées par du paraloïd B 72 dilué à $5 \%$ dans du chlorothène. Dessins et fiches techniques ont été faits. Ensuite, les plaques ont été encollées sur une gaze et une toile à l'aide de paraloïd B 72 dilué à $30 \%$ dans du chlorothène, afin que l'on puisse les retourner et égaliser le mortier de revers, le consolider avec un fixatif aqueux (le caparol) et une mince couche de mortier synthétique (composé de mowilith I), de mowilith D 025, d'eau, de sable et de poudre calcaire). Les bords ont été laissés libres pour tout collage postérieur éventuel.

L'ensemble étant bien sec, la gaze et la toile furent enlevées avec le solvant d'origine de la colle (chlorothène), puis les peintures furent de nouveau fixées au paraloïd à $5 \%$. Ces opérations de restauration terminées, il était possible de manipuler des plaques, très fissurées et fragiles à l'origine, et de tenter les collages en vue d'une restitution du décor.

L'observation du diagramme (fig. 1) fait apparaître deux groupes de peintures dont les mortiers sont différents; l'un a servi de support à l'autre, il lui est donc évidemment antérieur (cf. n $\mathrm{n}^{0}$ F. 16 bis).

a) Les peinlures les plus anciennes: le groupe 1 .

Il est essentiellement connu par les fragments F.16 et F.16 bis. Chaque fragment comporte trois couches distinctes : deux de mortier beige et une couche de surface blanche. De la décoration, nous ne connaissons qu'un fond blanc strié et une bande marron de $3,9 \mathrm{~cm}$ de largeur encadrée de deux bandes noires de 1,1 cm de large (cf. F.16). Ces premiers enduits ont été piquetés afin d'être recouverts par de nouvelles peintures : celles du groupe 2.

4 A. Barbet 'L. Testard-Mler.at, Rapport provisoire sur les peinlures murales de Famechon, Paris-Soissons, 1976, dactyl. 


\section{b) Le groupe 2.}

1. Les morliers: le mortier des peintures du groupe 2 est, lui aussi, constitué de trois couches distinctes, comme l'attestent les fragments ayant conservé leur épaisseur maximale $\left(2,2 \mathrm{~cm}\right.$, cf. diagramme : F.29). La troisième couche ${ }^{5}$ est un mortier de couleur crème dont la composition est à base de chaux et de sable et qui contient parfois des éléments assez grossiers : les particules de sable peuvent atteindre $0,1 \mathrm{~cm}$ de diamètre et la chaux forme parfois des nodules. Nous avons remarqué quelques traces de paille (long. maximum observée : $3 \mathrm{~cm}$ ). Cette couche de mortier n'excède jamais $1,2 \mathrm{~cm}$ d'épaisseur et était appliquée sur les peintures piquetées du groupe 1 (cf. supra). La deuxième couche est une couche de mortier de tuileau, rose, caractéristique du groupe 2 , dont les éléments constituants ne dépassent pas $0,7 \mathrm{~cm}$ de diamètre. L'épaisseur de cette couche est variable et peut atteindre jusqu'à $1,8 \mathrm{~cm}$ (cf. diagramme : F.6). La couche de surface est blanche. Elle est composée d'un lait de chaux contenant de la calcite. Son épaisseur est de $0,2 \mathrm{~cm}$. Dans la plupart des cas, il ne reste que les deux premières couches, la rupture s'étant opérée entre le mortier rose et le mortier de couleur crème.

2. Les tracés préparatoires et les empreintes: les tracés préparatoires n'ont été observés que sur deux fragments : F.1 et F.17 (fig. 7). Sur F.1, il s'agit d'un tracé à la cordelette donnant une direction horizontale. Cette dernière est recouverte d'un filet jaune séparant la bande noire supérieure d'un champ noir au-dessus de l'entablement. Sur F.17, c'est une dépression large de $0,5 \mathrm{~cm}$ qui prépare un arc de cercle séparant un champ rouge d'un fond blanc marbré de vert clair (fig. 9). Cette dépression est recouverte d'une guirlande de perles bleues et noires.

Enfin, le mortier du fragment F.6 présente une dépression arrondie de $2 \mathrm{~cm}$ de diamètre et de $0,8 \mathrm{~cm}$ de profondeur maximale (fig. 3). La peinture la recouvre parfaitement et aucun raccord n'est visible. De toute évidence, ce trou a été fait accidentellement dans le mortier frais, avant la pose de la pellicule picturale semble-t-il. Peut-être s'agit-il d'une perche d'échafaudage qui a heurté par accident l'enduit encore mou?

3. La pellicule picturale: elle a été très attaquée par l'humidité et l'acidité de la terre. Seuls les fonds ont été parfaitement appliqués et ils font corps avec l'enduit. Dans les autres cas, les coups de pinceau sont visibles. Leur largeur moyenne est de $0,5 \mathrm{~cm}$, sauf sur F.9 et F.10 où elle atteint $1 \mathrm{~cm}$. La peinture des blancs marbrés de vert (cf. F.17, en particulier) semble avoir formé une pâte épaisse dans laquelle les poils des pinceaux ont laissé des traces profondes (fig. 8 et 9). F.19 présente des traces de restauration ou de repentir puisqu'un champ bleu apparaît sous un jaune écaillé. En principe, un lait de chaux est étendu lorsqu'il y a une seconde décoration. Les pigments utilisés sont assez nombreux : on compte quatre jaunes différents, trois marron, quatre rouges, deux violets, deux bleus, deux verts auxquels il faut ajouter du rose, du crème, du blanc et du noir.

L'unité de technique (groupe 2) et de la palette employée nous incite à penser que

5 Par convention, la lecture des couches se fait à l'inverse de leur disposition chronologique, par conséquent, la troisième couche a été la première posée; cf. les fiches techniques mises au point par A. BARBET, Bulletin de liaison du C.E.P.M.R., no 4, Paris, 1979. 


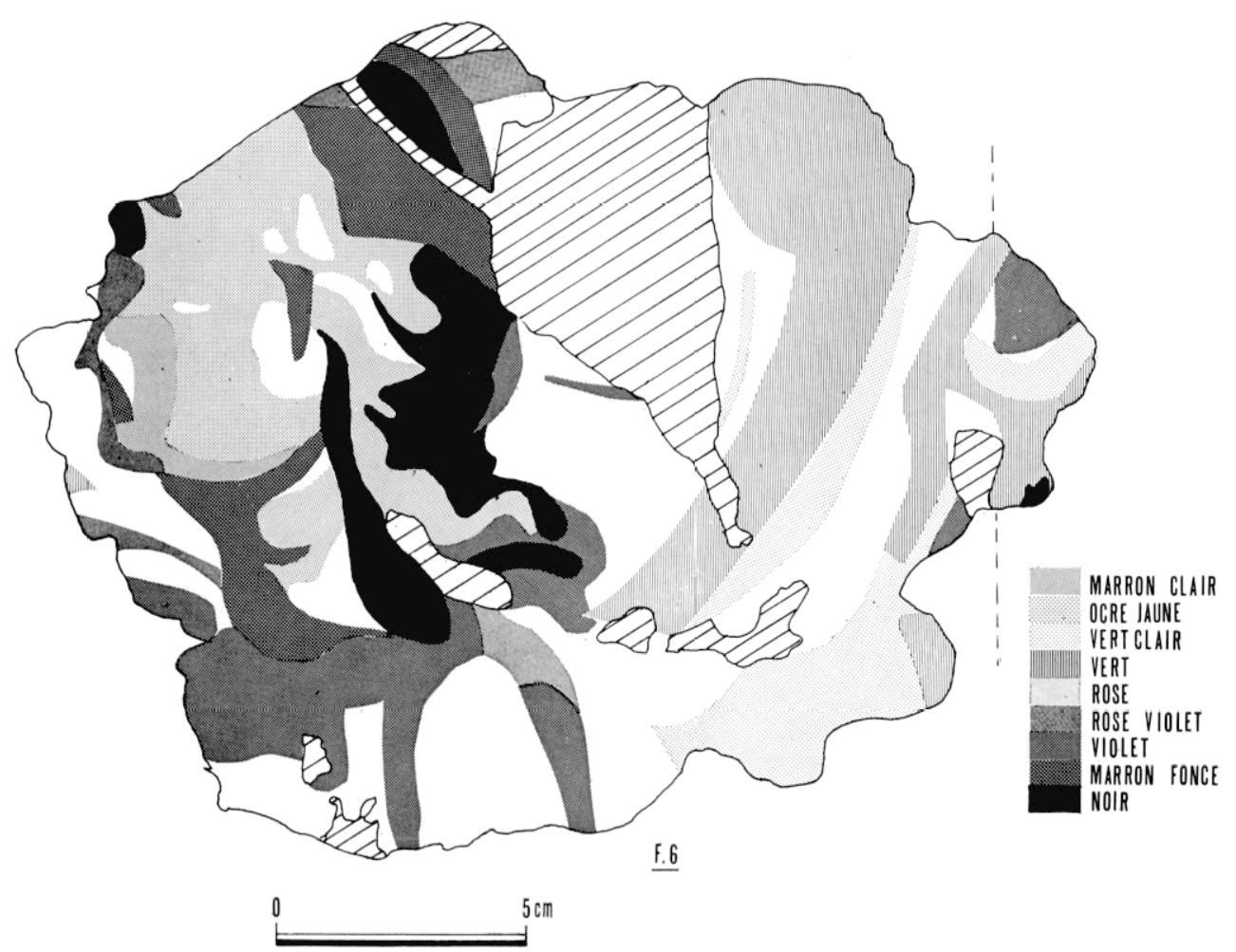

2 Figure ailée de Famechon.

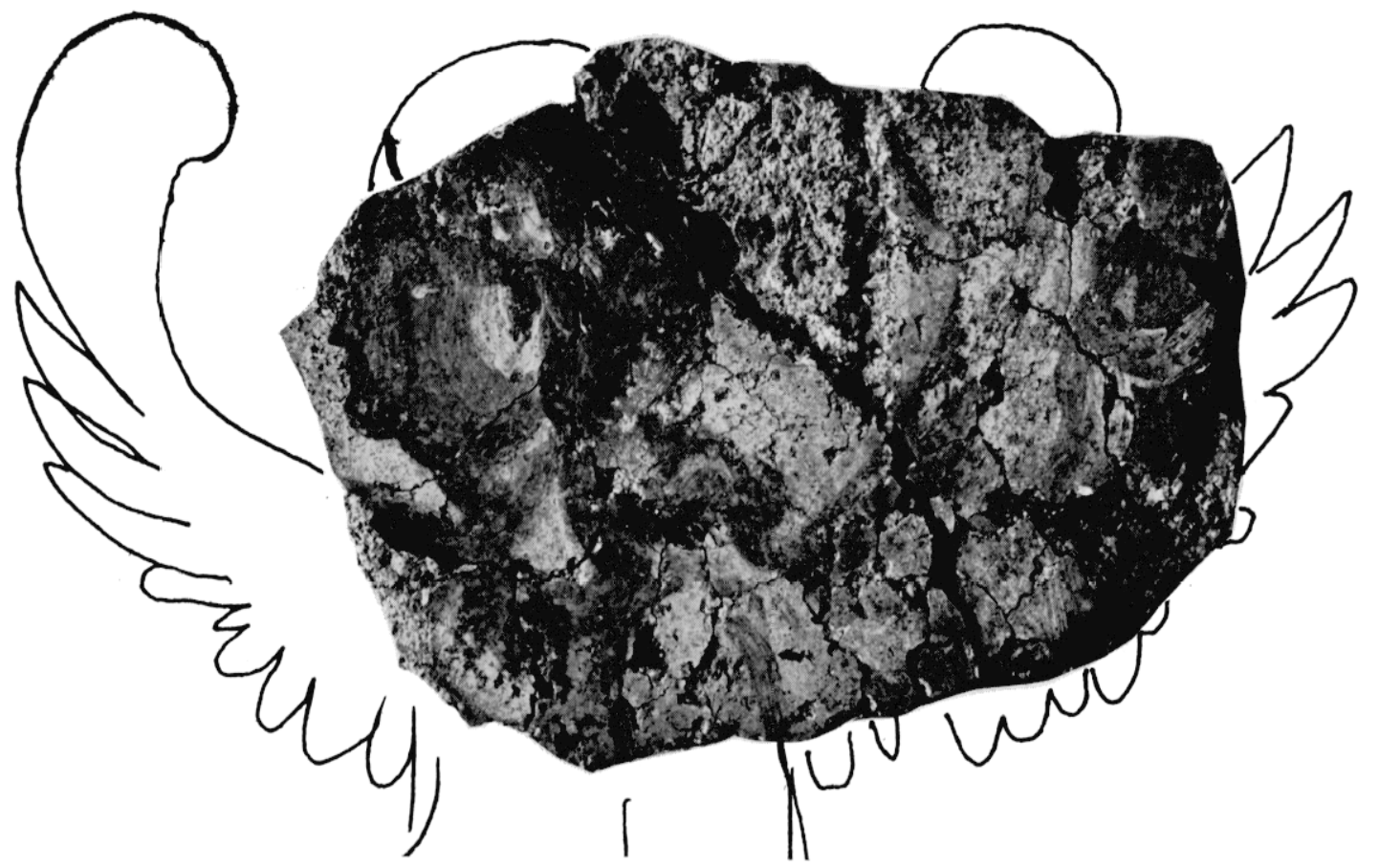

3 Figure ailée de Famechon. 


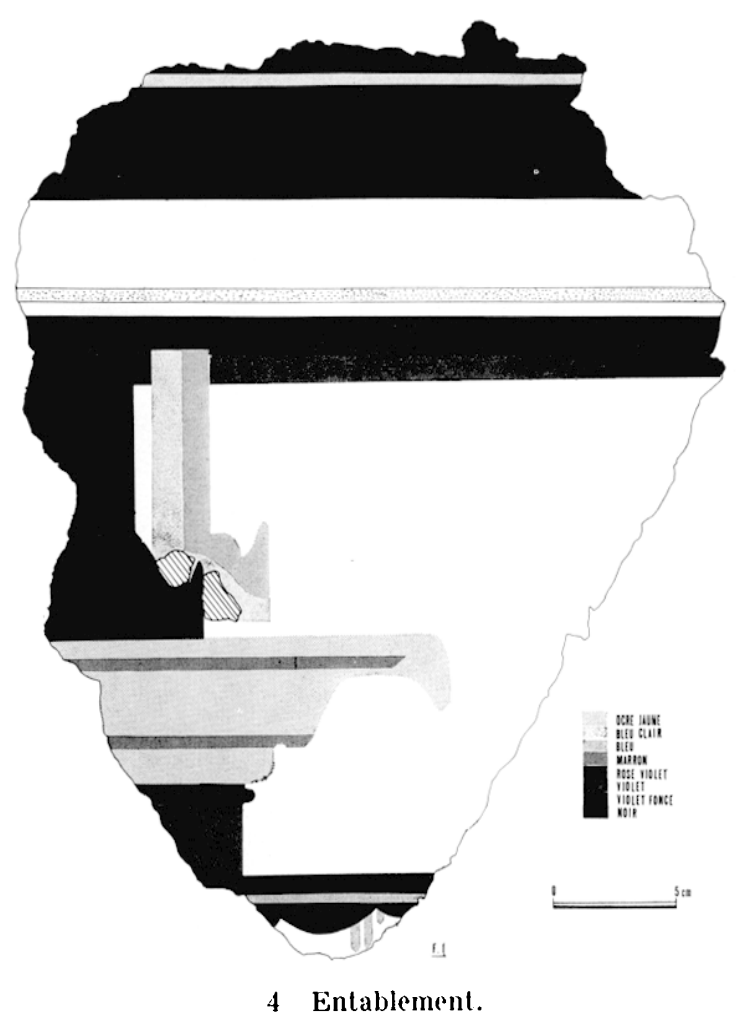

nous sommes en présence de fragments issus de la décoration d'une seule pièce que nous allons tenter de restituer. Cette restitution ne saurait cependant être globale car certains liens manquent entre les différents ensembles décoratifs qui se dégagent de notre étude.

\section{Description des peintures el inlerprélation.}

1. Le premier enscmble: figure féminine ailée (fig. 2 et 3). Le fragment F.6 est une pièce remarquable : il représente une tête de personnage ailé féminin, de profil à gauche, qui se découpe sur un fond uni blanc. Un filet rouge foncé dessine le visage, qui a été travaillé en jaune, blanc et noir, le modelé étant indiqué par la disposition des taches de couleur. Les cheveux tombent sur le cou, noirs avec des reflets jaunes. Des filets roses dessinent les bras. Le haut du bras droit porte des indications de modelé et d'ombres par le renforcement du rose et par l'emploi du noir. Le bras gauche a été peint linéairement en rose plus clair. Sur la droite, le départ des ailes, en coquille, se fait en vert et vert clair, donnant ainsi une impression de profondeur. A l'extrême droite du fragment, on voit le départ d'un motif composé d'une verticale rose, que les ailes recouvrent partiellement. La présentation de profil du personnage, ainsi que ses ailes en coquille suggère une sphinge (fig. 11, en haut).

2. Le deuxième ensemble: entablements (fig. 4 à 7). On peut en avoir une idée assez précise grâce à six fragments importants : F.1 à F.ó et F.9. Le morceau clé est le fragment F.1, de $35 \mathrm{~cm}$ de hauteur sur $29 \mathrm{~cm}$ de largeur : c'est lui qui nous permet l'identification de l'ensemble et qui nous donne toutes les séquences. Ces entablements sont surmontés 

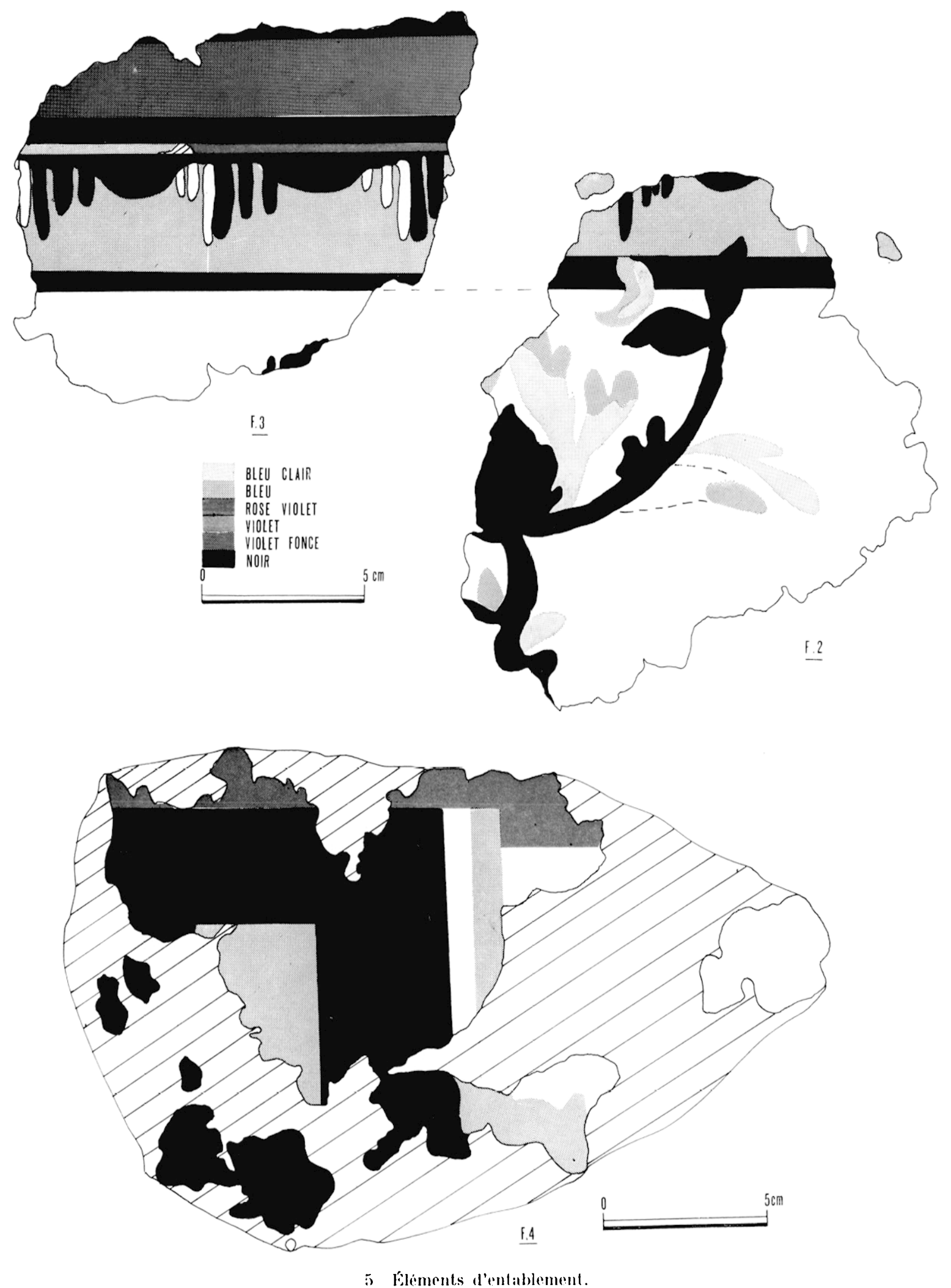

5 Elements dentablement. 

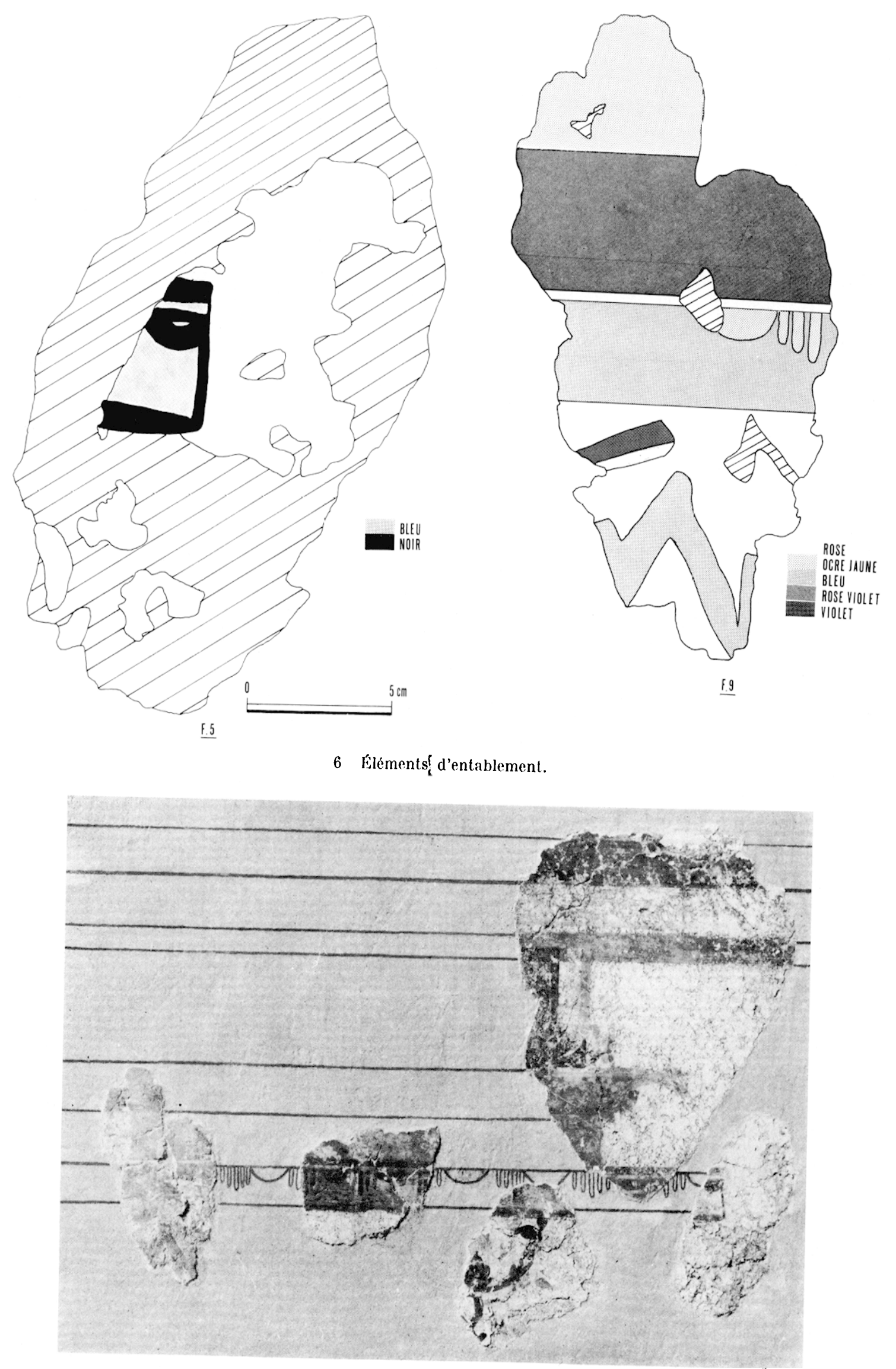

7 L'entablement reconstitué. 
d'un bandeau blanc - qui n'est autre que le fond sur lequel ils se découpent - et d'un champ noir divisé par un trait jaune. L'architrave est violette et violet clair. Le fragment F.4, autre extrémité droite d'entablement (fig. 5, en bas), identique à celle de F.1 (tronqué), montre le découpage de la première frise noire en caissons bleus. Les extrémités droites ont leur ombre portée peinte en bleu et en bleu clair (cf. F.1 et F.4) (fig. 5 et 6). Au-dessous se dessine une corniche très débordante, à moulures horizontales marron, dont l'extrémité retombe fortement en bec arrondi. Une deuxième frise violette surmonte un motif proche de celui des perles et des pirouettes, noires et blanches. F.3 nous montre une séquence plus longue de ce motif, dont F.5 est une des extrémités droites (fig. 5). La présence d'ombres portées bleues (cf. F.1, F.2, F.4) indique que la lumière provient de la gauche, et nous permet ainsi de placer les différents éléments les uns par rapport aux autres dans l'ensemble de notre restitution. Sous l'entablement s'accroche une sorte de console végétalisée noire, aux ombres portées bleues et bleu clair (cf. F.2). F.9 pourrait être interprété comme un fragment de nœud et de ruban flottant (fig. 6, à droite).

3. Le troisième ensemble: fond marbré vert et noir (fig. 8 et 10). Les fragments les plus représentatifs sont F.8, F.15 et F.39. Ils montrent un fond vert marbré de noir, entouré d'un bandeau ocre-jaune, de $5,5 \mathrm{~cm}$ environ de largeur, encadré de deux traits noirs. Le tout est surmonté d'un champ blanc (cf. F.8 et F.39). F.15 nous donne un angle, ce qui permet de supposer un caisson carré ou rectangulaire. Nous pensons qu'il s'agit de fragments de plinthes.

4. Le qualrième ensemble: losange sur fond ocre jaune (fig. 9). Les fragments F.10 et F.10 bis offrent un champ ocre jaune sur lequel s'inscrivent deux angles de $45^{\circ}$ dont les côtés violets ont une largeur de $5,8 \mathrm{~cm}$. Là encore, nous pensons qu'il s'agit de fragments appartenant à une plinthe, que l'on peut restituer comme un losange dans un caisson rectangulaire.

5. Le cinquième ensemble: champ rouge el fond blanc marbré. Cet ensemble est représenté par quatre fragments : F.12 et F.12 bis, F. 17 et F. 17 bis. Un grand champ rouge en arc de cercle est séparé d'un fond blanc marbré de vert clair par une guirlande d'ovales bleus et noirs. Étant donné l'importance du rayon du cercle, cet ensemble peut prendre place à l'intérieur d'un caisson de panneau ou de plinthe.

6. Divers: fragments de peinlures murales à champ vert prédominant. F. 11 et F.35 ont en commun une bordure rose violet. F. 36 et F.37 présentent chacun un angle droit et sont bordés d'un bandeau jaune à traits blancs et noirs : ils font peut-être partic de la plinthe. F.38 est différent par son vert plus clair. Quant à F.7, il présente, au-dessus du champ vert, des bandes plus ou moins horizontales de différentes couleurs. A noter, dans sa partie supérieure, un angle de couleur grise. Bien que ces fragments soient de dimensions relativement importantes, rien ne nous permet de les intégrer dans la restitution globale.

Essai de restitution (fig. 10). Nous avons établi cette proposition de restitution à partir de l'ensemble des entablements, qui a pu être reconstitué en fonction de témoins matériels, et qui offre des garanties de stabilité dans la structure du décor. Nous avons la certitude qu'il y avait au minimum deux entablements, puisque des fragments de deux extrémités 

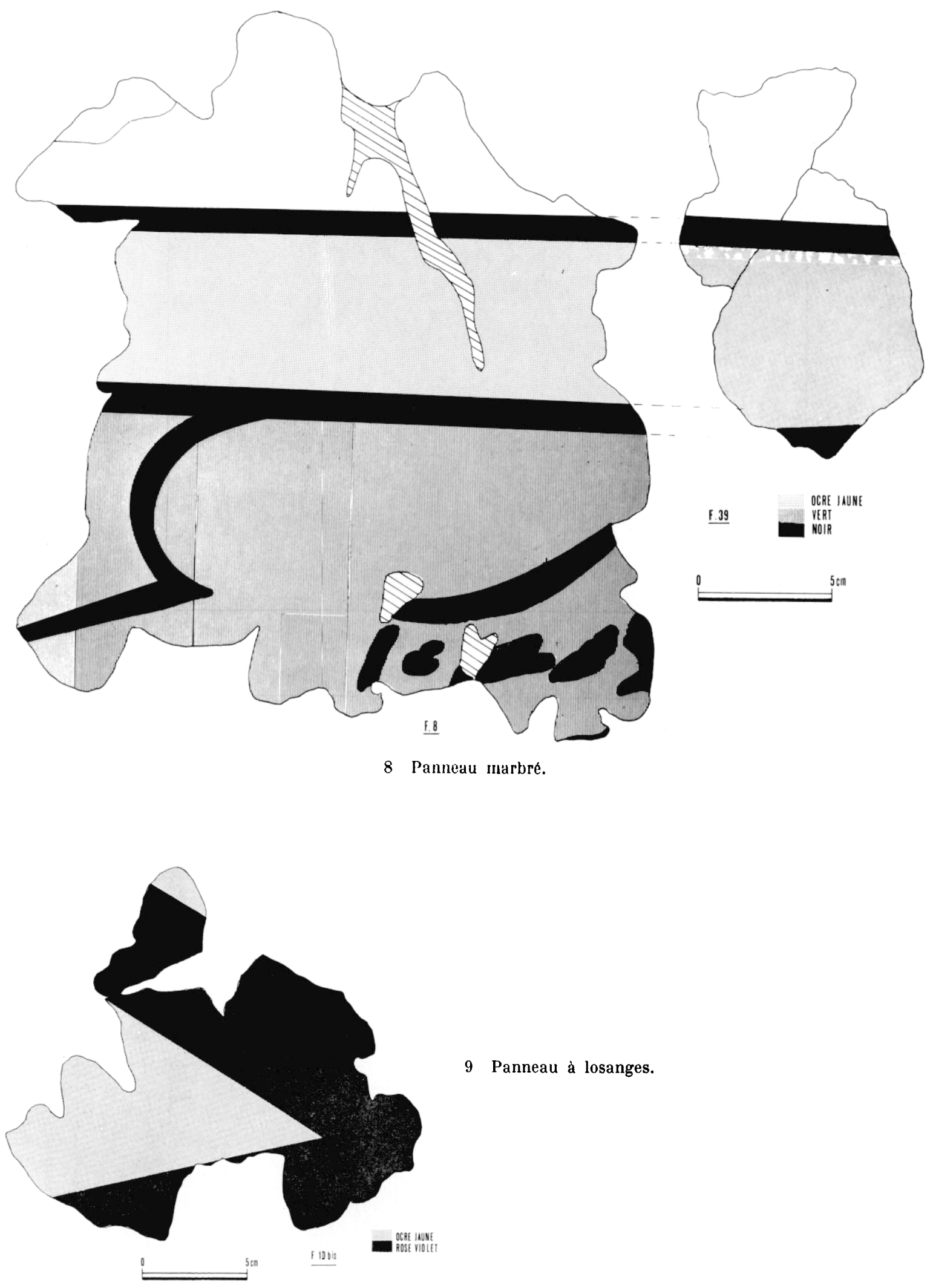


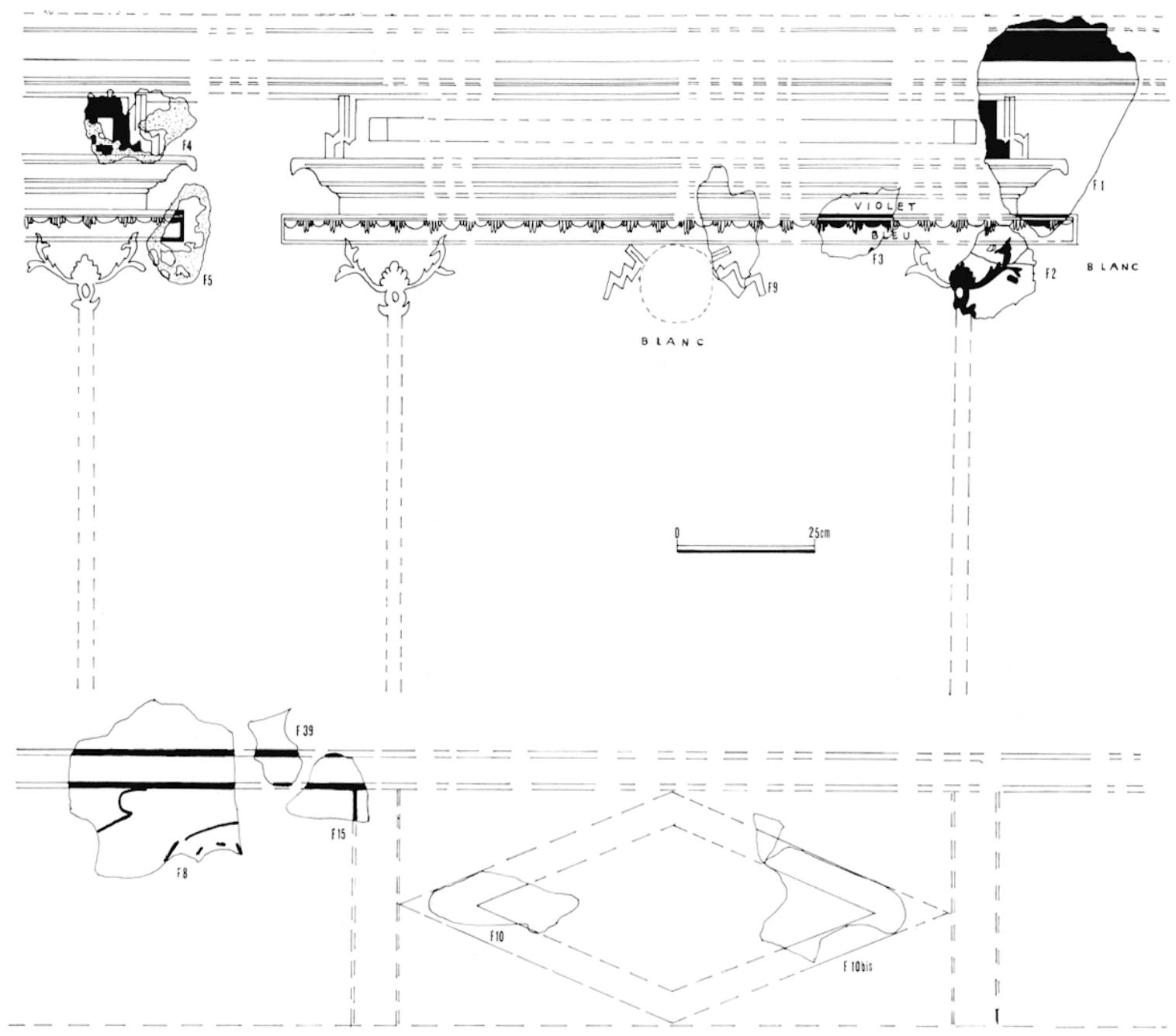

10 Restitution d'ensemble des peintures de Famechon.

droites (F.1 et F.4) ont été retrouvés. F.1 est suffisamment important pour permettre la reconstitution complète de l'ensemble. Le seul problème est posé par F.9, qui représente un fragment de nœud avec un ruban flottant. Nous l'avons restitué avec un clipeus au centre de l'entablement - bien que nous n'ayons aucune preuve de l'existence d'un clipeus - parce que c'est un poncif de la peinture romaine.

Nous avons placé en plinthe l'ensemble 3 (champ vert marbré de noir) et l'ensemble 4 (losange). En effet, fonds marbrés et décors géométriques sont courants en plinthe dans la peinture romaine. L'association des deux types de plinthe est possible ${ }^{6}$. La présence d'un champ blanc (cf. F.8 et F.39) permet un raccord logique avec les entablements.

L'emplacement de la figure ailée (ensemble 1) est beaucoup plus difficile à saisir. Aucun raccord matériel n'est visible. L'incertitude est telle que nous avons cru ne pas devoir l'introduire dans le schéma de restitution général.

\section{Didier VermeErsch.}

6 Cf. la pièce d de la maison des Vettii à Pompéi (VI, 15, 1). 

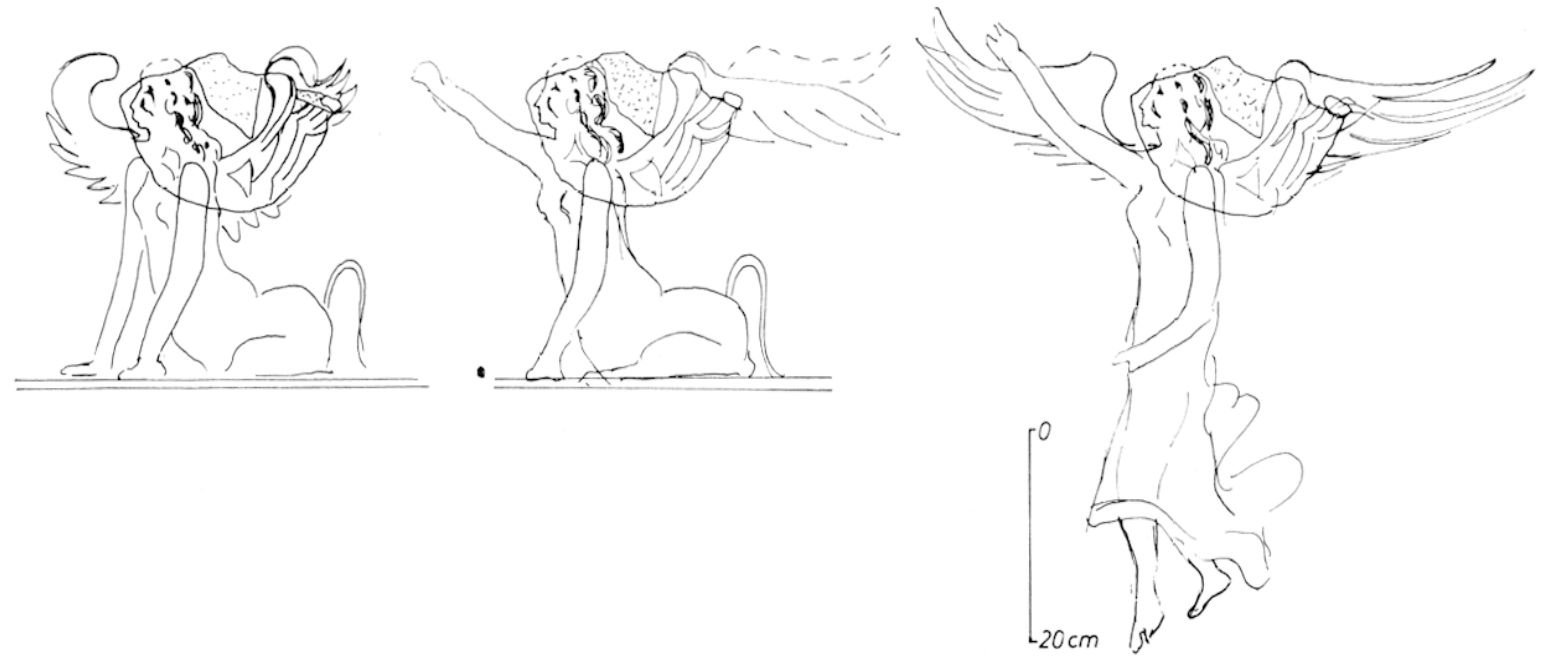

11 Restitutions graphiques de la figure ailée.

\section{II. Étude stylistique}

L'étude stylistique des peintures de Famechon est particulièrement délicate à mener car il s'agit de décors réellement fragmentaires dont la restitution reste largement hypothétique. Les liens entre les six ensembles différents qui ont été repérés sont difficiles à établir, faute de preuve, et supposent une ampleur du décor, impossible à mesurer. Toutefois, il n'est pas inutile d'explorer certaines pistes de recherche, pour situer la peinture dans la production romaine et tenter une approche de la datation.

A) La figure ailée ( $n^{0} F .6$ ).

A part la grande plaque qui nous conserve la tête, le départ des épaules et une partie des ailes, nous connaissons de minuscules fragments qui sont peut-être le bout des pattes. Le profil exact des ailes est difficile à restituer : une aile verte, concave, faisant un coude, est nettement lisible. Est-ce le bout arrondi d'une aile en coquille ou l'attache de grandes rémiges d'une aile éployée? Par ailleurs, on ne sait si la forme concave, à l'arrière, est bien la deuxième aile ou l'épaisseur de la première, car il y a un léger espace blanc entre les deux : peut-on supposer deux ailes repliées ensemble de profil à droite (comme nous l'avons restitué sur la figure 11, en haut à gauche)? Cependant il y a également une petite pointe de vert, au-dessus du bras droit, qui pourrait être interprétée comme la trace de l'autre aile (fig. 11, en bas). La lecture des traces pose donc un problème d'interprétation qui influence évidemment la restitution générale.

La position du bras droit est également conjecturale car son départ n'est pas clair sur le fragment. Repose-t-il à côté de l'autre bras (fig. 11, en haut à gauche) position fréquente pour les sphinges accroupies? Cela est douteux car le départ d'une trace rose, oblique, au-dessous du menton, indiquerait un bras levé ou tendu. La ligne est bien droite et non pas arrondie comme pour l'épaule gauche plus complète. 
Il subsiste une grande incertitude sur l'interprétation même de la figure. Le visage est intéressant, avec son nez fin, son menton aigu qui contraste avec la joue pleine et le cou un peu gras, dont une légère courbe indique une certaine mollesse. Les cheveux bruns semblent dénoués, en boucles souples sur les épaules. Aucun bijou n'est visible au cou ou aux oreilles et, si la facture picturale est très réussie, le type dépeint ne nous aide pas pour l'interprétation. La figure, selon la restitution globale qu'on en fait, mesurait entre 30 et $50 \mathrm{~cm}$.

Si l'on cherche dans la peinture murale romaine des figures avec des ailes de profil, on constate qu'une grande majorité des exemples appartient à des sphinges ailées juchées sur des corniches qui, nous allons le voir, ont eu un grand succès dans les provinces de l'Empire. En revanche, les figures de victoires ailées se présentent en général de face, les ailes déployées?. L'exemple d'une victoire de la Schola armaturorum à Pompéi nous incite cependant à la prudence car la tête est de profil et les ailes déployées font un coude arrondi à l'attache des grandes plumes, comme le schéma qu'on pourrait restituer à Famechon.

Sphinge ailée, génie ou victoire? de la solution de ce problème dépend en partie la place qu'il faut attribuer à la figure. Si nous étions en mesure de le faire nous pourrions choisir entre une sphinge ailée, au-dessus de la corniche conservée, ou une victoire volant au milieu d'un panneau. Le fond blanc, autour de la figure, ne peut nous aider, car il existe aussi bien autour de la corniche F.1 qu'autour de F.3 et de F.4, c'est-à-dire latéralement et au-dessus de l'entablement. Cependant la corniche noire supérieure est suivie d'un champ noir incomplet, ce qui interdit de placer une sphinge juste au-dessus (fig. 4).

Sphinges dans la peinture romaine: le motif, bien connu dans l'art funéraire grécoromain, apparaît en guise d'acrotère dans la peinture du II ${ }^{\mathrm{e}}$ style, dans la villa dite de Poppée à Oplontis (Torre Annunziala) vers 40 av. J.-C. La figure est vue de face, les bras reposent sur la corniche moulurée dont le profil est couronné d'une palmette. Les ailes sont déployées de face et le corps semble celui d'un lion; il est de profil, tourné vers l'extérieur du bâtiment qu'il couronne ${ }^{8}$ (fig. 12). On notera les coups de lumière vifs sur les joues, l'épaule droite, les seins.

$\mathrm{Au}$ troisième style, le motif est rendu de façon hiératique, comme cette sphinge accroupie, immobile, au coin d'un entablement suspendu dans le vide, dans la maison dite de la Colonnade Tuscanienne à Herculanum9 (fig. 13). L'aile est rigoureusement de profil et cache celle qui est derrière, l'avant-train n'est pas humain. La datation de cette pièce semble encore soumise à discussion mais on avance fréquemment la date du milieu du $\mathrm{I}^{\mathrm{er}}$ s. ap. J.-C.

Avec le IVe style et le renouveau d'architecture variées, les sphinges ailées peuplent en abondance les sommets des corniches, qu'elles partagent avec toutes sortes d'autres êtres fantastiques, comme des griffons, et des monstres marins divers, des tritons. L'exemple

7 Cf. les figures de victoires ailées de la Schola, dans Spinazzol.A, Pompei alla luce degli scavi nuovi di via dell' Abbondanza (1910-1923), Rome, 1953, fig. 174 à 177.

8 A. De Franciscis, La villa romana di Oplontis, dans Neue Forschungen in Pompeji, Recklinghausen, 1975, p. 9-38.

9 M. ManNi, Le pillure della casa del Colonnalo Tuscanico, dans Monumenti della piltura antica scoperti in Italia, III, Ercolano, 2, Rome, 1975. Voir également dans la Villa Impériale, à Pompéi, cité par $\Lambda$. Linfert, dans Römische Wandmalerei der nordwesllichen Provinzen, Cologne, 1975, pl. 7. 


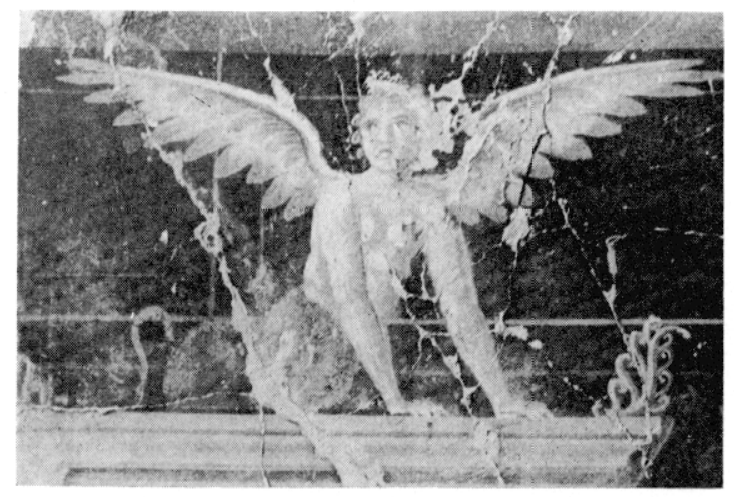

12 Sphinge de la villa de Poppée à Oplontis, triclinium no 14 .

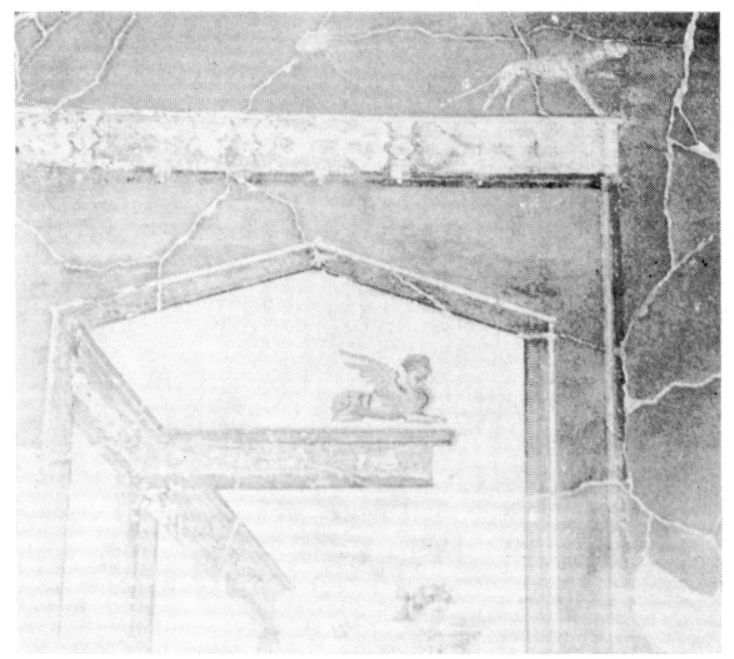

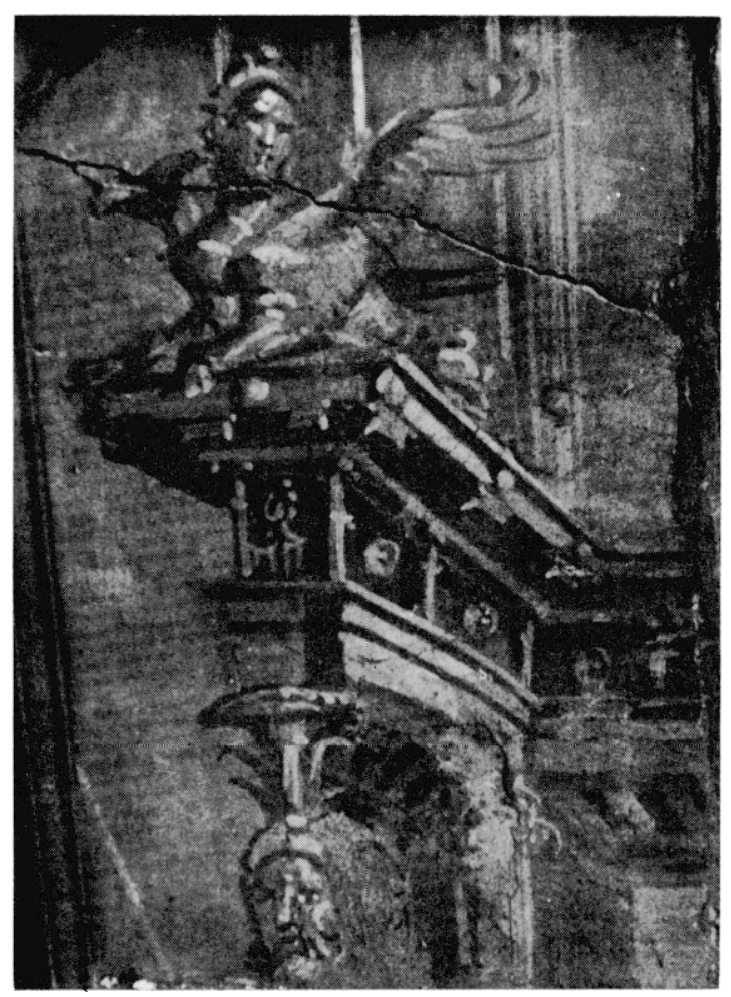

14 Sphinge des réserves du Musée national de Naples.

13 Maison à la Colonnade Tuscanienne à Herculanum, pièce $n^{\circ} 3$.

que nous donnons, des réserves du Musée National de Naples, montre l'utilisation de la lumière en traits violents, destinés à donner l'impression du luisant sur des surfaces polies : marbre ou métal (fig. 14). La sphinge n'a d'humain que la tête, elle est vue de trois-quarts et elle est couronnée d'un diadème ${ }^{10}$.

Si nous regardons la peinture provinciale aux $\mathrm{I}^{\mathrm{er}}$ et $\mathrm{II}^{\mathrm{e}} \mathrm{s}$., on s'aperçoit du succès $\mathrm{du}$ thème, utilisé aussi bien en sommet de candélabre qu'au-dessus d'un entablement. Audessus d'un candélabre on citera celui du Musée de Trèves, de la Gilberstrasse: assise sur le train arrière, sur l'ombelle du candélabre, de face, violemment éclairée, les deux bras posés se terminent en pattes et en griffes ${ }^{11}$.

Récemment d'autres décors similaires ont été découvert à Gujasseix, commune de Rougnat (Creuse). Les figures sont montées sur une corolle de canthare sans anse, posé

10 Voir d'autres sphinges ailées, dans Roux et Barré, Herculanum et Pompéi, Paris, 1840, I, pl. 17 ; K. Sch efold, Vergessenes Pompeji, Berne-Munich, 1962, pl. 85-86, maison des Vettii (VI, 15, 1), pièce e ; pl. 78, maison de Pinarius Cerealis (III, 4, 4), sphinges en acrotères, bras tendu pour tenir des guirlandes, ailes de profil ; pl. 36, caserne des gladiateurs $(\mathrm{V}, 5,3$ triclinium mur ouest, sphinges minuscules bras levé, de profil.

11 C. P. SteIner, Römische Wandmalerei in Trier, dans Trierer Zeitschrift, 2, 1927, p. 62, fig. 14 et photothèque du Landesmuseum de Trèves. 
sur une corniche, sous laquelle pend un bouclier d'amazone ${ }^{12}$. Vues de face, accroupies, les ailes déployées, elles correspondent à l'iconographie habituelle en Italie. Elles portent des armilles aux bras.

On peut trouver aussi ce type de figure sur des entablements; c'était, semble-t-il, le cas sur une peinture inédite des réserves du Muséc de Trèves, provenant de la basilique. Sur une bande verte, se détachant sur un fond rouge, comme à Cujasseix, une sphinge accroupie de face, aux ailes déployées, appuie une de ses pattes, au bras orné de deux armilles (au poignet et près de l'épaule) sur la bande. La tête n'est pas conservée, exceptée une frange où l'on reconnaît des cheveux.

A Cologne, dans les fouilles exécutées du côté sud du dôme, toute une paroi peinte écroulée a pu être ramassée et recomposée. Le mur A nous propose un schéma utile pour des comparaisons. Des colonnettes à chapiteaux corinthiens encadrent des panneaux rouges et soutiennent un entablement plat orné d'oves et couronné aux angles de sphinges ailées qui alternent avec des cygnes. Entre les panneaux rouges les bandes intermédiaires sont occupées par des candélabres très riches et par des scènes de vendanges pour l'espace central de la paroi, plus large ${ }^{13}$ (fig. 15). Les sphinges ailées nous montrent un profil, rarement gracieux, une coiffure en chignon (fig. 16). Le corps est massif, les membres antérieurs ressemblent plus à des pattes qu'à des bras, et le peintre, qui a voulu représenter les ailes de profil étendues, n'a pas su rabattre convenablement l'aile arrière, qui est plaquée et ramenée vers l'avant, devant la poitrine. Le fond est noir, comme toute la frise. A. Linfert date ce décor du début du ${ }_{1}{ }^{\mathrm{e}} \mathrm{s}$, , en raison de ressemblances stylistiques avec une production provinciale qui commence déjà à la seconde moitié du $\mathrm{i}^{\mathrm{er}} \mathrm{s}$. ap. J.-G.

Sur le mur G, aux angles des entablements, se dressent des figures ailées de profil, dont les deux bras sont levés sans qu'on puisse préciser le geste car la peinture est cassée à cet endroit. Le corps se termine de façon monstrueuse mais la photo qui en est donnée, dans la publication de A. Linfert, est trop exiguë et les explications trop succinctes pour qu'on puisse préciser mieux ${ }^{\mathbf{1 4}}$.

A part la position en sommet de candélabre ou d'entablement, les sphinges, comme tout le cortège des êtres ailés monstrueux, peut servir de vignette au centre d'un panneau uni. D'échelle généralement réduite, elles alternent avec des griffons, des aig̣les et des cygnes. On citera pour mémoire, à Pompéi, les sphinges accroupies sur des panneaux verts de la maison dite de l'Éphèbe, d'un troisième style qui était très élaboré (Pompéi, I, 7, 10), les pégases et les sirènes de la maison de la Petite. Fontaine (VI, 8, 23) ${ }^{15}$.

Pour la Gaule, nous avons l'exemple unique, donné par A. Blanchet dans son Réper-

12 Cf. J.-Y. AutexıE, J.-F. Fı.Écıten, Les peinlures murales d'une villa romaine en Limousin, dans Archeologia, 85,1975, p. 38.41 et couverture.

13 C. A. Linfert, Römische Wandmalereien aus der Grabung am Kölner Iom, dans Kölner Jahrbuch 13, 1972-1973, p. 65-75; pl. 19, l pour la restitution d'ensemble.

14 A. L.INFERT, Römische Wandmalerei der nordwestlichen Provinzen, Cologne, 1975, pl. 36.

15 Pour la maison de l'Éphèbe, voir l'aquarelle de F. el A. Niccolini, Le Case ed $i$ monumenli di Pompei, Naples 1854-1891, II, pl. LXX, qui conserve la totalité du décor dont il ne subsiste plus actuellement que des lambeaux. Pour la maison de la Petite Fontaine, voir W. $\mathrm{Z}_{\mathrm{AuN}}$, Die schönsten Ornamente und merlwürdigsten Gemälde aus Pompeji, Herculanum und Stabiae, Berlin, 1828-1852, II, 5. 


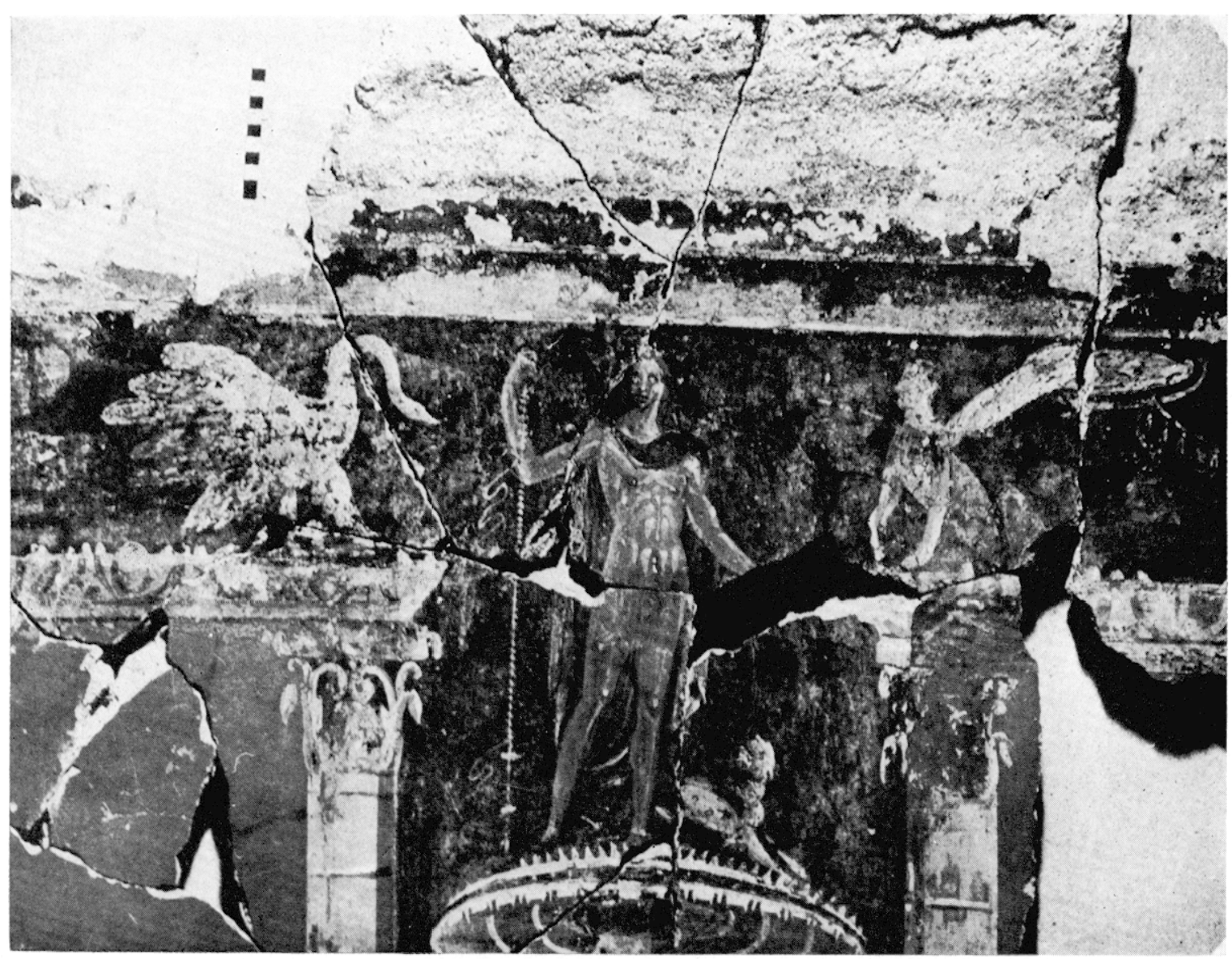

15 Cologne, restitution graphique du mur A, côté sud du dôme.

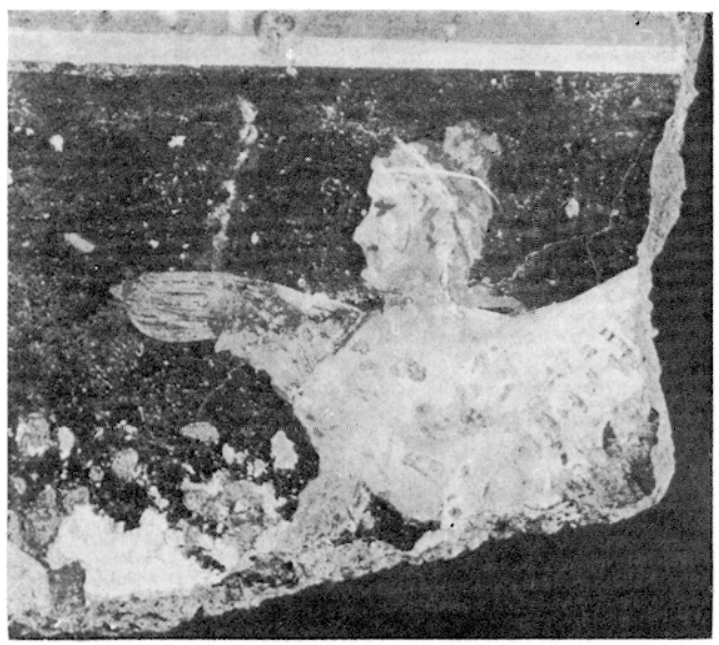

16 Cologne, détail d'une sphinge ailée de l'entablement, mur B. 
toire, d'un griffon posé sur un nuage et se détachant de profil sur un panneau bleu, en provenance de Bouzemont dans les Vosges ${ }^{16}$.

$\mathrm{Au}$ vu de ce panorama des figures de sphinges, on constate qu'on en trouve en grande majorité en guise de couronnement, de profil ou de face, ailes déployées ou de profil, plutôt statiques, mais qu'il existe des figures qui flottent sur un fond uni de panneau médian. A part quelques rares cas où la figure lève le bras; généralement pour tenir une guirlande (cf. Cologne, Pompéi) les sphinges sont au repos. En revanche les figures de victoires et de génies volants ont des mouvements de bras fréquents et variés.

Figures volantes ailées dans la peinlure provinciale: nous avons dit, déjà, l'extrême fréquence des figures ailées dans la peinture pompéienne et post-pompéienne et noté la rareté de l'attitude de profil. Nous l'avons trouvé cependant à la Schola Armaturorum à Pompéi. La mode s'en est répandue très tôt en Gaule et nous en connaissons plusieurs témoins. Les peintures murales de la rue Saint-Thomas à Strasbourg, étudiées par R. Forrer, montrent, sur un fond noir, un génie ailé nu, de face, dont les ailes arrondies font plutôt penser à celles d'un éros qu'à une figure d'adulte ${ }^{17}$. Il faudrait pouvoir vérifier sur l'original, ce détail.

De même les peintures murales de la Villa d'Ancy (commune de Limé, Aisne) restituées sur l'album Caranda, offrent, côte à côte, une figure de femme volant, un voile au-dessus de la tête et un jeune homme ailé soufflant dans un instrument ${ }^{18}$. La restitution ne donne pas les morceaux réels et l'aquarelle présente bien des anomalies; de plus, la figure est présentéc de face, sur un fond rouge et n'a pas grand-chose à voir avec la nôtre.

A Vaison-la-Romaine, dans les fouilles récentes faites derrière la cathédrale, sur le fond d'un panneau rouge, on a pu reconstituer la figure entière d'un génie ailé, portant une palme et un vase doré ; il est vu de profil mais la forme des ailes, déployées, n'est pas très lisible ${ }^{19}$. Sur un autre panneau se trouvait une femme nue, ailée, également sur fond rouge. Le corps et la tête sont de face, le regard incliné vers le bas, l'aile est courte, tombante, le bras gauche est relevé au-dessus de la tête. Comme on le voit, le schéma diffère sensiblement de celui de Famechon.

Il existait sûrement d'autres peintures présentant de telles figures, et certaines d'entre elles sont encore inconnues, en cours de reconstitution (à Narbonne, Clos de la Lombarde) ou dorment dans des réserves.

On mentionnera, en dernier lieu, une figure ailée au sommet d'un candélabre sur fond noir, qui se présente de face, coiffée d'un diadème, provenant de Vienne et qui est en cours d'étude ${ }^{20}$. Le modèle diffère, mais il atteste le goût pour ce type de personnage dans la peinture en Gaule.

Si l'on tente de résumer les critères à retenir pour interpréter la figure de Famechon, en fonction de l'enquête menée, on s'aperçoit qu'ils sont tous incertains : la vue de profil

16 Cf. A. Blanchet, Élude sur la décoralion des édifices de la Gaule romaine, Paris, 1913, pl. I.

17 R. Forrir, Strasboury-.1ryentorate, Strasbourg, 1927, II, pl. CXXV el pl. LVI.

18 F. Morfau, Colleclion Caranda, Saint-Quentin, 1877-1388; livres $11^{\mathrm{e}}$ et $12 \mathrm{e}$ pl. 90.

19 Cf. F. Salviat, Informalions archéologiques, dans Gallia, 35, 1975, p. 536, fig. 25.

20 A. Barbet, dans A. Barbet, A. Canal, J. Lancha, A. Pelletter, Iécouvertes archéologiques récentes $a$ Vienne (Isère), $3^{\mathrm{e}}$ partie, article à parâ̂tre dans Monuments et Mémoires Fondalion E. Piot, 1980. 
existe aussi bien pour des Victoires que pour des sphinges, même si elle est plus courante pour ces dernières ${ }^{21}$. La forme des ailes, en coquille ou déployées, importe mais on ne peut assurer qu'elles soit bien parallèles sur la figure de Famechon. Les coups de lumière violents sur la joue tachée de jaune évoquent les reflets métallisés, qui sont peut-être plus fréquents pour les sphinges, mais cela n'est pas certain. Enfin, se livrer à une étude comparée des échelles et des dimensions des différentes catégories n'aboutirait pas car il y a beaucoup de variétés. Il est donc plus sage de ne pas conclure, bien que notre opinion personnelle penche plus volontiers vers l'hypothèse d'une sphinge.

\section{B) L'entablement.}

Il nous est mieux connu dans son profil que dans son développement longitudinal. A la base, une sorte d'architrave violette et bleu clair, limitée par un trait noir à la partie inférieure, et par un filet également noir à la partie supérieure, d'où pendent des traits noirs ou blancs, par trois, et de longueur décroissante, entre des demi-ovales noirs (cf. $n^{\circ}$ F.3) (fig. 5). On peut interpréter ceci comme l'ombre portée de perles et de pirouettes, plutôt que celles d'oves et de fers de lance. En effet l'ovale est couché, les traits pendants seraient un peu trop longs pour des fers de lance.

Cette architrave était très saillante par rapport à la corniche qui la surmonte, comme le prouve la grande plaque F.1 (fig. 4). Nous savons qu'elle s'arrêtait selon un profil droit (cf. F.5). La corniche est ocre jaune, à traits marron pour simuler les creux et son profil exagère considérablement la doucine supérieure qui retombe un peu comme un bec arrondi. Les singularités se poursuivent sur la frise bleu à cadre noir, au moins aux extrémités car il n'y a pas assez d'échantillons conservés pour savoir s'il s'agissait d'un décor à métopes et triglyphes (fig. 7). La base de la frise, à son extrémité, est relevée en angle aigu, après un arrondi et l'ombre portée en bleu clair dessine le même profil en avant du cadre noir. On comprend mal l'origine de cette forme (cf. F.1 et F.4). Enfin au-dessus, une mince architrave, qui devait être continue, montre une double bande, rose violet et violette.

Les points de comparaison sont malaisés car les bizarreries de ces architectures sont évidentes et il est douteux de trouver les mêmes, alors qu'il s'agit d'une peinture provinciale, qui a peu de parallèles. L'exagération du profil de la corniche ne se retrouve pas à Cologne et si nous comparons avec une peinture du $\mathrm{II}^{\mathrm{e}}$ style, comme la corniche sous la sphinge ailée de la villa de Poppée à Oplontis (fig. 12), on mesure la différence. Cependant sous la sphinge de la maison à la Colonnade Tuscanienne (fig. 13), la corniche est très saillante par rapport à l'architrave.

Il y a tout de même un parallèle frappant à établir avec une peinture fragmentaire de la région parisienne : dans la villa de Guiry-Gadancourt (Yvelines), dite des TerresNoires, et publiée par P.-H. Mitard, on trouve les mêmes ovales sombres sous une architrave, en alternance avec des traits blancs et sombres, groupés par trois, en dégradé2 ${ }^{22}$. L'aquarelle qu'en a donnée J. Sirat montre une gamme de bruns, différente de celle de Famechon,

21 Sur 31 victoires répertoriées par S. Reinach, six seulement sont de profil ; cf. S. Reinach, Réperloire des peintures grecques et romaines, Paris, 1922, p. 146, 1 ; 146, 8 ; 146, 4; 146, 9 ; 149, 4 ; 149, 6.

22 P.-H. Mitard, La villa gallo-romaine de Guiry-Gadancourt, dans Gallia, 16, 1958, p. 266-280, fig. 8 et 9. 
mais on y trouve la même particularité des traits blancs par trois, avec des traits marron, en alternance avec ces ovales qu'on a rencontré nulle part ailleurs. Il s'agit du même processus de déformation d'un motif d'origine architecturale, que nous avons supposé être des perles et pirouettes, et donc d'une même sphère d'influence artistique, pourrait-on penser.

Au-dessus de cette architrave prenait place un monstre ailé, aux ailes de profil, à la patte avant ornée de deux bracelets, l'un au poignet, l'autre à l'avant-bras, ce qui rend tout à fait improbable la restitution d'un griffon. Nous aurions là plutôt l'image d'une sphinge accroupie ${ }^{23}$ car si on n'a jamais vu de grifion avec des bracelets, nous pouvons mentionner de nombreux exemples de sphinges aux bras chargés d'armilles (cf. Cujasseix, Cologne). L'intérêt de cette nouvelle interprétation est de renforcer encore la comparaison qu'on peut établir avec Famechon et l'hypothèse de la sphinge échafaudée à son propos. Le même fond blanc rapproche les deux séries ${ }^{24}$.

Pour les autres ornements il est difficile de proposer des comparaisons. Il en est ainsi pour les éléments de losanges mis en plinthe, qui n'ont aucun filet d'encadrement, aucun effet spécial qui permettent de les rattacher à un groupe éventuel ${ }^{25}$. De même les imitations de marbres qui sont assez traditionnelles et qui font penser à un système avec imitation d'opus seclile, qui eut un grand succès en Gaule ${ }^{26}$.

Du point de vue du soin de l'exécution, nous avons déjà remarqué le bon traitement de la figure ailée qui fait regretter la perte du reste. De même l'entablement suppose une décoration riche, puisqu'on n'avait pas hésité à utiliser un cadre architectural assez complexe.

Peut-on se risquer à proposer une datation? Ne pas le faire serait plus prudent, pour éviter des spéculations hasardeuses ultérieures, néanmoins il semble qu'on puisse risquer le $\mathrm{II}^{\mathrm{e}} \mathrm{s}$. D'une part les éléments de datation directe nous orientent vers la fin du $\mathrm{II}^{\mathrm{e}} \mathrm{s} . \mathrm{au}$ plus tard, puisque les débris de peinture de Famechon faisaient partie d'un dépotoir daté de cette époque (comme celui de Guiry), d'autre part les comparaisons établies avec la

23 A noter que P.-H. Mitard mentionne des moitiés de visages humains p. 274, alinéa $e$ de l'article de Gallia (cf. note précédente). Peut-être certains d'entre-cux pourraient-il convenir pour des sphinges.

24 En ce qui concerne la datation de la fosse no 13 , P.-H. Mitard me communique les renseignements suivants, issus du rapport de fouilles : le matériel céramique jeté en même temps que les peintures date en majorité du II $^{\mathbf{e}} \mathbf{s}$. Des sigillées ornées sont assignées à l'époque de Trajan-Hadrien (type Déch. 490-Osw. 842, Déch. 20-Osw. 25, Déch. 344-Osw. 638) à l'époque d'Hadrien et d'Antonin (type Déch. 1037-0sw. 2239) et à l'époque d'Antonin (type Déch. 734-Osw. 1374). Les céramiques communes moins typées, moins faciles à dater, donnent pour l'une d'elles, une jatte carénée en terre grise, une époque peut-être tardive, si on se fonde sur les typologies anciennes (III ${ }^{\mathrm{e}} \mathrm{IV}^{\mathrm{e}} \mathrm{s}$.). Il semble que ce dépotoir ail servi durant le III $^{\mathrm{e}} \mathrm{s}$. Les peintures seraient donc au plus tard contemporaines du matériel céramique jeté au rebut en même temps qu'elles, soit du ${ }_{1}{ }^{\mathrm{e}} \mathrm{s}$. si on se fonde sur la date des céramiques sigillées.

25 Comparer avec d'autres plinthes à losanges plus typés; cf. Périgueux, villa de la rue des Bouquets, F. Coupry, Informations archéologiques, dans Gallia, 1961, p. 380, fig. 18 a et b, et Ribemont-sur-Ancre, cf. $\Lambda$. Quit.LET, Les enduits peints du sancluaire gallo-romain de Ribemont-sur-Ancre, dans Bulletin de la Sociélé des Anliquaires de Picardie, 1974, p. 287-314, repris dans Lalomus, 37, 2, 1978, p. 361-363.

26 Pour la question de l'imitation de marbre dans la peinlure romaine, voir II. Eristov, Corpus des fauxmarbres peints à Pompéi, dans Mélanges de l'École Franģaise de Rome, Anliquilés, 91, 1979, 2, p. 693-771, suile à l'exposé théorique : Un algorilhme appliqué à la classification des imilations de marbre dans la peinlure pompéienne, dans MEFRA,'88, 1976, 2, p. 705-717. Voir pour l'extension aux provinces, L. Abad Casal., Las imilaciones de "crustae " en la pintura mural romana en España, dans Archivo Español de Arqueologia, 50-51, 1977-1978, nos 135-138, p. $189-208$. 
peinture campanienne et avec la peinture provinciale indiquent une période qui va de la fin $\mathrm{du} \mathrm{I}^{\mathrm{er}} \mathrm{s}$. au début $\mathrm{du} \mathrm{II}^{\mathrm{e}} \mathrm{s}$.

De toute évidence le thème a été populaire durant le ${ }_{I^{\mathrm{e}}}{ }^{\mathrm{e}} \mathrm{s}$. et la combinaison des éléments fournis par la chronologie interne des sites, les lerminus ante quem des fosses et dépotoirs avec ceux fournis par le style, une fois qu'ils pourront être affinés par d'autres enquêtes, devraient permettre de préciser mieux la période exacte de production. Le lien entre les peintures de Famechon et celles de Guiry n'est pas négligeable et s'explique parfaitement par la proximité des deux villas, éloignées à moins de $100 \mathrm{~km}$ l'une de l'autre.

Grâce aux soins compétents de l'équipe de fouille de Famechon, et malgré le caractère très parcellaire de ce décor, nous disposons d'un jalon utile pour la connaissance de la production de la peinture romaine au nord de la Gaule.

\section{Alix Barbet.}

N.B. Provenance des illustrations : M. V. Mieze et D. Vermeersch, fig. $1,2,4$ à 6,8 à 10 ; $\mathrm{A}$. Barbel, fig. 3,7 , 11 ; H. Eristov, fig. 14; fig. 12, d'après A. de Franciscis, dans Neue Forschungen in Pompeji, Recklinghausen, 1975, fig. 20 ; fig. 15 et 16, d'après A. Linfert, dans Kölner Jahrbuch, 13, 1973, pl. 19, 1 et 24, 2. 